\title{
El PAPEl multifuncional de los modelos EN LA CIENCIA Y SU GRAN PODER INFERENCIAL ${ }^{1}$
}

DAIRON ARBOLEDA ${ }^{2}$

Las grandes leyes clásicas (gravitación, electromagnetismo...) permiten edificar modelos cuya exactitud numérica desafía la imaginación (...) pero desde que se sale del dominio -relativamente estrecho- en el que estas leyes se aplican sin pérdida, la situación se degrada rápidamente.

René Thom (1982)

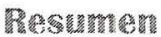

El objetivo fundamental de este artículo es el de resaltar la multiplicidad de funciones que desempeñan los modelos en la ciencia y, sobre todo, sus grandes virtudes para inferir aspectos relacionados con el mundo real. Defenderé la tesis de que más que artefactos epistémicos, componentes de un sistema distribuido cognoscitivo o de medios de representación, los modelos deben concebirse como la principal herramienta para inferir y predecir el comportamiento de sistemas reales.

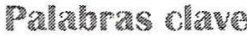

Modelo, Teoría, Representación, Semántica, Isomorfismo, Lingüista, Similitud, Estructuralismo.

1 Este artículo hace parte de un trabajo más amplio relacionado con el papel de los modelos en la ciencia y su relación con las leyes y teorías.

2 Ingeniero Civil de la Universidad Nacional de Colombia. Especialista en Gestión Energética Industrial en el ITM y candidato a doctor de la Universidad del País Vasco. Investigador del Grupo Da Vinci del ITM. 
Alusinare

The main goal of this article is to stress the multiplicity of functions that the science models carry out, and above all, their great virtue to infer aspects related to world. I'll defend the theory about the more explanations, distributed cognitive components of a system or representation ways, the more conceived the models as the principal tool to infer and predict the behavior of real systems.

Way worthes

Model, Theory, Representation, Semantic, Isomorphism, Linguist, Similitude, Structuralism. 


\section{WTRZDUUCCLÓN}

A pesar de que los modelos han sido utilizados por los científicos desde hace varios siglos, para representar fenómenos relacionados con el mundo real (leyes de Kepler y de Newton por ejemplo); sólo a mediados del siglo XX se les ha tratado de concebir como componentes básicos de las teorías científicas.

En la primera parte de este trabajo, analizo brevemente los antecedentes fundamentales acerca de las distintas concepciones sobre la estructura de una teoría científica y el papel de los modelos en la concepción semántica o modelo-teórica, que es la de mayor vigor en la actualidad.

Posteriormente, abordo la discusión sobre el papel de los modelos como representación de sistemas reales, discutiendo algunas percepciones al respecto y defendiendo la tesis de que "El valor representacional de un modelo, se mide por su poder y fecundidad para predecir e inferir el comportamiento de un sistema real", sin necesidad de acudir a criterios de similitud o isomorfismo, como lo han propuesto algunos autores.

Al final del trabajo, defiendo la tesis de que los modelos no se pueden abordar desde una sola perspectiva, dada la gama amplia de modelos que existen, sus distintos significados epistemológicos y las múltiples funciones que desempeñan en la ciencia. Los modelos pueden ser usados para representar porciones del mundo real, como artefactos epistémicos, en el sentido de que podemos obtener conocimiento de ellos y sobre todo, como herramientas para predecir el comportamiento de variables físicas, sociales o económicas.

Desde esta concepción, este artículo hace parte de un trabajo más amplio relacionado con los modelos científicos y que aborda cuestiones de interés:

- Herramientas básicas utilizadas en la construcción de modelos. Se explica la importancia de las técnicas estadísticas, el análisis dimensional, el concepto de proporcionalidad y las ecuaciones diferenciales entre otras herramientas. 
- Determinación de variables físicas a partir de modelos conocidos y validados empíricamente.

- Distintas visiones acerca del concepto de ley natural.

- Las cláusulas Ceteris Paribus (lo demás permanece constante, las otras variables tienen incidencia nula) en la formulación de modelos científicos.

- Una propuesta didáctica para la enseñanza de las ciencias básicas a partir de la formulación y construcción de modelos, como elemento esencial para la formación de investigadores.

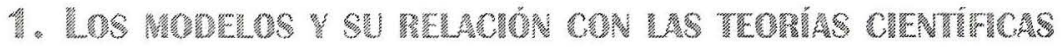

Cuando se aborda la pregunta acerca de lo que es una teoría científica, como mínimo debe analizarse los tres enfoques clásicos: la concepción lingüística, el enfoque historicista y la visión extralingüística o semántica.

La que se ha denominado comúnmente como concepción heredada, tuvo gran vigor después de la segunda guerra mundial y hasta los años 70. Sus representantes más significativos fueron Carnap, Reichembach, Hempel y Nagel entre otros. En este enfoque sintáctico o lingüista se conciben las teorías científicas como cálculos axiomáticos abstractos conectados a la experiencia y a la observación directa.

Como conectores Carnap utiliza reglas de correspondencia, Reichembach definiciones coordinativas y Hempel enunciados interpretativos.

$\mathrm{Al}$ igual que los representantes de la corriente historicista, no estoy de acuerdo con este aparato axiomático descontextualizado de la realidad que viven las comunidades científicas. Además del valor pragmático de los modelos y teorías, es preciso tener en cuenta sus limitaciones prácticas, las abstracciones, idealizaciones y aproximaciones utilizadas en su construcción; aspectos no considerados en la concepción lingüista.

El enfoque historicista critica el hecho de que en la concepción enunciativa no se consideren casos concretos de la historia de la 
ciencia en el análisis de las teorías científicas. El máximo representante de esta corriente, es el filósofo y físico estadounidense Thomas Kuhn con su obra "Estructura de las revoluciones científicas" (1962). Otros filósofos de esta vertiente son Imre Lakatos y paul Feyerabend. Cabe resaltar la importancia de Willard V. Quine en lo que se ha llamado el giro naturalista como crítica a la concepción enunciativa.

"Quine, por su parte, es el principal impulsor reconocido de lo que se ha llamado el giro naturalizado en la filosofia de la ciencia, giro comúnmente caracterizado por el rechazo del análisis conceptual a priori como genuino método filosófico" (IBARRA/MORMANN, 1997, pág. 20).

Contrario a la corriente historicista, el enfoque semántico no critica el uso de métodos formales en la concepción enunciativa, sino el uso de una lógica primitiva e inadecuada ${ }^{3}$.

Para el trabajo que yo pretendo realizar, no considero pertinente profundizar en las concepciones ligüísticas e historicistas y en adelante me centraré en las vertientes semánticas, que a pesar de que tienen enfoques diferentes utilizan herramientas matemáticas diversas (topología, teoría de conjuntos, análisis no Standard...), coinciden en que el concepto de modelo es central en las teorías científicas; caso contrario en la concepción heredada.

El gran eslabón de las concepciones semánticas lo inicia Patrick Suppes a mediados del siglo XX, que inspira el nacimiento del estructuralismo metateórico de Joseph D. Sneed y Wolfgang Stegmuller en los años 70 y del empirismo constructivo de Bas van Fraassen en los años 80 (DIEZ / MOULINES, 1999). Luego se adhieren a esta corriente Ronald Giere y Frederick Suppe en Estados Unidos. En la actualidad y dentro de la gran gama de enfoques semánticos diferentes se destacan Newton Da Costa, Steven Fren-

3 DíEZ y MoUlines. Fundamentos de la filosofia de la ciencia. Barcelona: Ariel, 1999, en el capítulo ocho realizan un buen análisis de la concepción axiomática con ejemplos muy concretos. 
ch, Octavio Bueno, Mauricio Suárez, Nancy Cartwright, Morrison y Morgan, entre otros.

En resumen, para todas las vertientes semánticas, "Las teorías científicas, más que sistemas de enunciados, consisten en sistemas de modelos".

Como el objetivo de mi trabajo es el de resaltar las virtudes de los modelos en el avance de la ciencia (no sólo de la física) y no como éstos se estructuran a las teorías científicas, obviaré la matemática utilizada por los estructuralistas y autores como Bas van Fraassen, Octavio Bueno, Da Costa y French. Se acoplan mejor a mi propuesta, las cuestiones tratadas por Giere, Suárez y Cartwright. Ibarra y Mormann ${ }^{4}$ porque realizan un amplio análisis de la concepción de la representación de van Fraassen.

Como conclusión de este breve recorrido acerca de las concepciones básicas sobre la estructura de las teorías científicas, se pueden destacar los siguientes puntos:

- Aunque la concepción heredada se considera superada, no puede ignorarse. Díez y Moulines ${ }^{5}$ realizan un análisis sobre los puntos básicos de esta visión enunciativa.

De igual manera, la siguiente frase enmarca el pensamiento de uno de los grandes inspiradores de este enfoque:

En la matemática no se sabe de que se habla ni si lo que se dice es verdad. (Russell).

Einstein, acorde con esta visión señala:

Cuando las proposiciones matemáticas se refieren a la realidad no son ciertas; cuando son ciertas, no hacen referencia a la realidad (1921).

4 IBARRA, Andoni y MORMAnN, Thomas. Representaciones en la ciencia de la invariancia estructural a la significatividad pragmática. Barcelona: Ediciones del Bronce, 1997. $320 \mathrm{p}$.

5 DíEZ y MOULINES. Fundamentos de la filosofía de la ciencia. 1999. p. 307, 308. explican las tres limitaciones básicas que presenta la concepción heredada y que hacen más viables las concepciones semanticistas. 
Como puede observarse, en la concepción heredada las proposiciones matemáticas son verdades lógicas, vacías de contenido cognoscitivo. La matemática carece de contenido fáctico, tal como lo expresa Carnap "La matemática no nos dice nada acerca del mundo que habitamos" 6 .

Diametralmente opuesta a esta visión reduccionista de la relación de la matemática con el mundo real, a lo largo de mi propuesta estaré enfatizando en la conexión significativa entre los modelos matemáticos, la realidad y el quehacer de los científicos.

- Contrario a la creencia de muchos, las corrientes semánticas han trabajado con más profundidad la historia de la ciencia y el papel de las comunidades científicas, que la vertiente historicista de Kuhn. Incluso, filósofos como Ronald Giere, han convivido varios años con los científicos, para tener una idea clara de qué es la ciencia y cómo se construyen las teorías científicas.

- Una gran ventaja de la concepción semántica es que permite hablar de ley científica ${ }^{7}$ involucrando la economía, la psicología o la sociología; y no exclusivamente de la ley física como ocurre en la concepción heredada. Por ejemplo, Nancy Cartwright ha trabajado significativamente en el campo de los modelos económicos y Ronald Giere ha utilizado herramientas de la neurociencia y la psicología cognitiva. Mi propuesta es prolífera en el empleo de conceptos estadísticos y leyes probabilísticas.

- Como lo señalan Hendry y Psillos, la concepción lingüista limita la representación de una misma teoría, utilizando modelos diferentes:

6 IV Coloquio internacional de filosofía e historia de las matemáticas. En: Ideas y valores, Universidad Nacional. Bogotá. Nos. 92 y 93. p. 80.

7 ECHEVERRÍA, Javier. Filosofía de la ciencia. Madrid : 1998. p. 75, 76. "Este giro permite emprender el análisis de teorías que ya no proceden exclusivamente de la física, como ha sido frecuente en buena parte de los filósofos de la ciencia del siglo XX”. p. 75,76 . 
"Una teoría debe identificarse con algo extralingüístico, que puede admitir formulaciones diferentes. ¿y qué otra cosa podría ser más apropiada que una estructura matemática abstracta? (Hendry y psillos, 1998, pág. 6).

Suppe cita como ejemplos las formulaciones equivalentes de la teoría cuántica (formulación matricial y mecánica de ondas) y la mecánica clásica de partículas en sus formulaciones Lagrangianas y Hamiltonianas. Ésta es una de las bondades de la concepción semántica o modelo-teórica.

Posteriormente, en este mismo trabajo, presentaré dos modelos probabilísticos totalmente diferentes que me permiten estimar el valor del número pi $(\pi)$.

- El hecho de que la concepción lingüística haya sido superada, no significa que la ciencia no necesita de componentes lingüísticos.

Como lo señala Ronald N. Giere (2004, pág. 1) definir la clase de modelos no es suficiente para decir algo sobre el mundo. Se requiere de algo lingüístico, una hipótesis teórica. Los científicos utilizan las similitudes entre modelos y aspectos del mundo real para formular hipótesis y generalizaciones.

\section{El PAPEL DE LOS MODELOS EN UA REPRESERTACHÓN

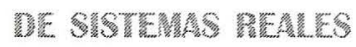

Las bondades de un modelo para representar sistemas reales, dependen, en gran medida, de los propósitos que se fija el usuario del modelo. Por ejemplo, si mi propósito al usar un mapa bidimensional de una ciudad es el de ubicar fácilmente cualquier sitio de ésta, se puede afirmar que el mapa es una excelente representación de la ciudad. Si, en cambio, quiero tener una idea sobre el tipo de edificaciones y de la estética de la ciudad, el mapa no tiene un valor representacional. Algo similar ocurre con la información adicional que puedo obtener de la maqueta de una casa, con respecto a la percepción que me brinda un simple plano de ésta en dos dimensiones. 
Como en la ciencia se pierde cualquier rasgo de subjetividad y los intereses trascienden del plano individual al colectivo; es bueno delimitar el concepto de modelo, dada la gran diversidad de modelos existentes y su multiplicidad de significados.

Como señala Giere, las cosas que comúnmente se denominan "modelo", parecen formar una clase bastante heterogénea que incluye a los modelos físicos, a los modelos a escala, a los modelos analógicos y a los modelos matemáticos. Mi propuesta, básicamente, se centra en los modelos matemáticos y probabilísticos. No consideraré la connotación del término modelo en el campo artístico o de la estética, ni las analogías usadas en las ciencias naturales. Yo utilizaré los modelos básicamente como una estructura matemática útil para representar, explicar e inferir aspectos del mundo real.

Para medir la fecundidad representacional de un modelo, se han utilizado diversos criterios: isomorfismo (Suppe, Fraassen), similitud (Giere), isomorfismo parcial (Da Costa y French). Mauricio Suárez (2003) argumenta la imposibilidad de reducir la representación científica a criterios de similitud o isomorfismo y propone una concepción inferencial de la representación. Sí $A$ representa a $B$, puedo inferir algo en $B$ investigando $A$ (Suárez, 2003). En la concepción de representación como isomorfismo: $\boldsymbol{A}$ representa a $B$ sí y sólo sí la estructura ejemplificada por $A$ es isomorfica a la estructura ejemplificada por $B$, donde se deben cumplix leyes de simetría, reflexividad y transitividad. Si $A$ es isomórfica a $B$, luego $B$ es isomórfica a $A$; cada estructura es isomórfica a sí misma; si $A$ es isomórfica a $B$ y $B$ a $C$, entonces $A$ es isomórfica a $C$ (Roman Frigg, 2002).

La crítica de Suárez a la vertiente estructuralista en su concepción de modelos como representación, radica en que la capacidad de un modelo de representar es inherente a él y no algo que se le agrega como un factor externo. Un modelo es una representación de un fenómeno y su utilización intencional no es una relación externa que nosotros podemos decidir agregar al modelo, pero es una parte esencial del mismo modelo. 
Ronald Giere ${ }^{8}$ propone el siguiente esquema, donde aparece la función representacional de los modelos:

$S$ usa $\mathbb{X}$ para representar $\mathbb{W}$ para un propósito $\mathbb{P}$

$\$$ : científico individual o comunidad científica

$W$ : aspecto del mundo real

$P$ : propósito que se persigue

X : modelos

A la pregunta: ¿Qué hace que los modelos sean aptos para representar aspectos del mundo?, Giere señala que la manera más importante, pero no la única, es aprovechando las similitudes entre el modelo y ese aspecto del mundo ${ }^{9}$.

En la línea de Suárez, a continuación presento dos ejemplos de modelos, que aparentemente no cumplen requerimientos de isomorfismo y similitud, sin embargo, puede demostrarse empíricamente que son buenas representaciones de la realidad. Aunque no son problemas relacionados con fenómenos físicos, sirven para corroborar mi tesis de que si un modelo tiene un buen poder predictivo, es un argumento suficiente para ser una buena representación del mundo real, independiente de cualquier otro criterio.

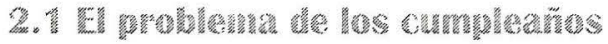

Este es un problema clásico que desafía la intuición y la realidad aparente.

¿Ha conocido alguna persona que cumpla años en el mismo día y mes que usted?

Tal vez esta situación no le haya sucedido, pero lo que sí con certeza ha encontrado, es que al menos dos de sus parientes o amigos cercanos cumplen años en la misma fecha.

8 GIERE, Ronald N. How models are used to represent reality. 2004. p. 2, 6.

9 GIERE, Ronald N. How models are used to represent reality. p. 2, 6. Suárez hace un análisis de este criterio en scientific representation: against similarity and isomorphism. 2003. p. 6. 
¿Apostaría usted, a que en un grupo de 25 personas al menos dos de ellas cumplen años el mismo mes y día? Por supuesto, que si es guiado por el sentido común no realizaría la apuesta. ¿Cómo es posible que en 365 casos factibles, pueda ocurrir tal coincidencia?

Se asombraría usted al saber que entre 25 personas, hay una probabilidad mayor al $50 \%$ de que al menos dos cumplan años la misma fecha; pero, más se sorprendería, al encontrar que entre 60 personas es casi seguro (99\%) de que ocurra este hecho.

La gente común se pregunta: ¿Cuál es la probabilidad de que una de las otras 59 personas cumpla años la misma fecha que yo?, pero lo que debería preguntarse es: ¿Cuál es la probabilidad de que cualquiera de los 60 cumpla años la misma fecha que cualquiera de los sesenta?

Cuando les planteé este problema a mis estudiantes de un curso de estadística, mostraron gran escepticismo ante los resultados mencionados, a pesar de que les deduje el modelo matemático que representa el fenómeno real ${ }^{10}$ :

Sea el evento $A=$ hay coincidencia de cumpleaños

$$
\begin{aligned}
& P(A)=1-\frac{{ }_{365} P_{n}}{365^{n}} \\
& n=\text { Número de personas } \\
& \text { un año }=365 \text { días } \\
& { }_{365} P_{n}=\frac{365 !}{(365-n) !}
\end{aligned}
$$

Para probar la fecundidad y el poder predictivo de este modelo, les propuse a mis 30 alumnos que simulara cada uno el fenómeno en el computador, generando 60 números aleatorios entre uno y trescientos sesenta y cinco. El uno representa el $1^{\circ}$ de enero, el 363 el 29 de diciembre y así los demás días del año.

10 SOTOMAYOR / WISNIEWSKI. Probabilidad y estadística para ingeniería y ciencias. 2002. p. 67, 68. Muestra una tabla con la probabilidad asociada a distintos valores de $n$ (número de personas) generada con el programa Excel. 
Si el modelo es una buena representación de la realidad, es de esperarse que la mayoría de los 30 estudiantes obtengan al menos dos números repetidos entre los 60 generados, como realmente ocurrió en el experimento.

Por encima de cualquier otro criterio, el poder inferencial y predictivo de este modelo, además de su consistencia con la teoría de las probabilidades, es lo que ocasiona que sea una buena representación de la realidad.

\subsection{Las ovaluaciones des escogencia multingle}

Si uno le propone a un grupo de estudiantes un examen de diez preguntas de escogencia múltiple, con cinco opciones cada una, de las cuales sólo una es la correcta, éstos tienden a sobrevalorar la probabilidad de aprobar el examen (al menos seis respuestas correctas) contestándolo aleatoriamente. Muchos creen, incluso, que la probabilidad de ganar la prueba es mayor del $50 \%$.

Antes de realizar una prueba de éstas en un curso de física, simulé este fenómeno con un grupo de 40 estudiantes, para desestimularlos en la selección al azar de la opción correcta. Ninguno de los 40 alumnos obtuvo como mínimo las seis respuestas correctas necesarias para aprobar el examen simulado.

El resultado obtenido era el que yo había inferido antes de entregar el cuestionario, apoyándome en la consistencia teórica y matemática del modelo binomial:

$$
\begin{aligned}
& P(x=k)=\left(\begin{array}{l}
n \\
k
\end{array}\right) p^{k} q^{n-k} \\
& k: \quad \text { número de éxitos (respuestas correctas) } \\
& n: \quad \text { número de ensayos (diez preguntas) } \\
& P=\frac{1}{5}: \text { probabilidad de éxito o de adivinar una respuesta } \\
& q=1-p=\frac{4}{5} \\
& \left(\begin{array}{l}
n \\
k
\end{array}\right)=\frac{n !}{(n-k) ! k !}
\end{aligned}
$$


De acuerdo con este modelo, la probabilidad de obtener seis o más respuestas correctas $P(x \geq 6)$, no llega siquiera al uno por ciento.

Realmente, no pretendo abordar todo el aparato matemático necesario para la realización de una crítica bien fundamentada del criterio de isomorfismo utilizado por los estructuralistas en la representación o el de similitud propuesto por Giere; he querido, más bien, mostrar cómo se puede constatar empíricamente el valor representacional de un modelo, desde criterios de inferencia y predicción.

\section{EL PAPEL MULVIFUNCIONAL DE LOS MODELOS gN LA CUECIA}

Es claro y es aceptado por la mayoría de filósofos de la ciencia, que la única función de los modelos no es la de representar aspectos del mundo real y sobre todo que no se trata de una simple relación diádica entre una entidad abstracta y una porción de la realidad; es necesaria la inclusión de la comunidad científica y el propósito para el cual se utiliza el modelo. Es decir, en la actualidad, los modelos han alcanzado un gran valor pragmático en todos los ámbitos de la ciencia. Van Fraassen expresa al respecto:

"La actividad científica es una actividad de construcción y no de descubrimientos: construcción de modelos que deben ser adecuados a los fenómenos y no descubrimiento de la verdad a cerca de lo inobservable" ( ).

Esta frase refleja la percepción del empirismo constructivo de Van Fraassen que tiene afinidades con el realismo constructivo de Giere ${ }^{11}$, en el sentido de que los científicos crean los modelos teóricos y no les son revelados directamente por la naturaleza. También los estructuralistas habían advertido la necesidad de agregar a la concepción modelo-teórica de Suppes una relación entre las estructuras matemáticas y las entidades exteriores ${ }^{12}$.

11 Se habla de realismo constructivo de Giere, porque la ciencia es una empresa dirigida a la obtención de teorías verosímiles o aproximadamente verdaderas. Los modelos son entidades abstractas construidas por humanos. Giere considera que las hipótesis afirman una similitud entre los modelos y los sistemas reales.

12 Fundamentos de la filosofía de la ciencia. 1999. Se realiza un análisis significativo de las distintas vertientes semánticas; sus afinidades y diferencias. 
Particularmente, me identifico con la propuesta de concebir a los modelos como artefactos epistémicos ${ }^{13}$, dado que éstos nos permiten obtener conocimiento, incluso de eventos que en un futuro van a ocurrir: el ingeniero hidráulico puede estimar los caudales y niveles de lluvia en una cuenca para los próximos años, como información fundamental para evitar sequías y que se ponga en riesgo a la población en una eventual inundación. El agente de seguros estima el valor por cobrar de una póliza, calculando el valor futuro estimado a pagar por ésta ${ }^{14}$. El administrador programa sus gastos e inversiones, basado en el nivel de ventas y utilidades que va a obtener el próximo año. En resumen, siempre nos quedaríamos cortos al tratar de resaltar con ejemplos concretos, las virtudes y fecundidad de los modelos científicos. Sin embargo, en este capítulo presento algunos casos donde se muestra el papel multifuncional de los modelos en la ciencia, sea concebidos como formas de representación, artefactos epistémicos o como mediadores entre la teoría y el mundo (Margaret Morrison, 1999); el papel de mediador lo puede realizar el modelo de varias formas: es mediador entre la teoría y el mundo cuando representa un sistema físico concreto, es mediador porque genera interrogantes al investigador y porque es una fuente de conocimiento de sistemas físicos limitados en su acceso para el hombre; como ocurre con los modelos cosmológicos y el modelo de quark de las partículas elementales ${ }^{15}$.

Una vez construido un modelo y validado como confiable y útil en la práctica por un método apropiado ${ }^{16]}$, se puede utilizar para diversos propósitos:

13 KNUUTILLA, Tarja propone concebir a los modelos como artefactos epistémicos en dos de sus artículos: a parser as an artefact: a material view on models y models, representation, and mediation.

14 El valor esperado de una variable discreta se calcula con la expresión $E(x)=\sum x p(x)$, donde $p(x)$ es la probabilidad asociada a cada valor que puede tomar la variable aleatoria $x$. En el caso de variables continuas se utilizan integrales para el cálculo de la esperanza matemática.

15 Models as representacional structures. p. 1.

16 El coeficiente de correlación ( $r$ ) es el criterio matemático utilizado comúnmente, para valorar como útil a nivel práctico un modelo de regresión. Mientras más se acerque 
- Inferir el comportamiento de una variable en el pasado o hacia el futuro. Presento el modelo utilizado para estimar la edad de un fósil por el método del carbono 14.

- Estimar el valor de una variable física como impedancia, constante de elasticidad de un resorte, aceleración de la gravedad o coeficiente de descarga entre un fluido y una superficie.

Con respecto a la utilización de los modelos, este trabajo presentó un método para estimar la aceleración de la gravedad en un punto cualquiera de la tierra o incluso en otro planeta.

- Estimar indirectamente el valor de un parámetro desconocido, comparando dos modelos distintos relacionados con un mismo fenómeno.

Presento un interesante método para estimar empíricamente el valor del número $p i$, usando la definición de probabilidad como frecuencia relativa ${ }^{17}$.

- Inferir el valor que tomará una variable dentro del rango experimental en el que se obtuvo el modelo.

Presento el ejemplo de un cultivo de bacterias, usando un modelo exponencial ${ }^{18}$.

$r$ al valor máximo de uno (100\%), más ajustado es el modelo a los datos empíricos. Un valor bajo de $r$ indica que el modelo no se debe usar como medio de inferencia.

17 La probabilidad de que ocurra un evento $A$ se puede calcular como $P(A)=\frac{n}{N}$ donde $n$ es el número de éxitos y $N$ el número de ensayos. Mientras más grande sea el valor de $N$, más se acerca $P(A)$ al valor real.

18 Un modelo exponencial de la forma $N=\alpha \beta^{t}$, se puede transformar a un modelo lineal utilizando logaritmos.

$\log N=\log \alpha \beta^{t}$

$\log N=\log \alpha+t \log \beta$

sustituyendo $\mathrm{y}=\log N, a=\log \alpha, x=t$

$\mathrm{b}=\log \beta$

se obtiene el modelo lineal

$y=a+b x$

Este método de linealización es útil en las ciencias experimentales. 


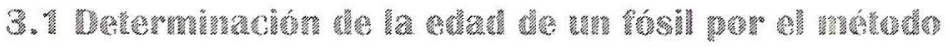

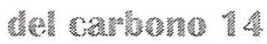

A mediados del siglo Xx, el químico Willard Libby inventó un método para calcular la edad de un fósil usando carbono 14. Por este modelo obtenido a partir de una ecuación diferencial lineal de primer orden, se le otorgó el premio Nobel de Física en 1962.

$$
A(t)=A_{0} e^{k t}
$$

$A_{o}$ es la cantidad inicial de carbono $14, A(t)$ es la cantidad de carbono 14 presente en un tiempo $t$.

El método se basa en que la semivida del C-14 es de 5600 años $t=5600 \Rightarrow A(t)=\frac{A_{0}}{2}$ Al reemplazar en la ecuación puedo determinar el valor de $k$.

Posteriormente, conociendo la cantidad presente de C-14 $A(t)$ de un fósil, puedo despejar el valor de $t$ reemplazando en el modelo propuesto por Libby.

Este modelo se utilizó para inferir la antigüedad del mobiliario de madera hallado en las tumbas egipcias y las envolturas de lienzo de los manuscritos del Mar Muerto. Aunque en la actualidad existen métodos más exactos para estimar la edad de un fósil, en su momento, este modelo causó impacto en las comunidades científicas.

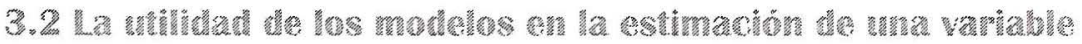

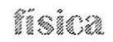

Posteriormente, presentaré varios ejemplos reales, en los que a partir de un modelo conocido se puede estimar una variable física que, en ocasiones, es muy dificil de medir directamente.

Se ha aceptado que para ángulos pequeños (menos de 20 ó 15 grados), el período de un péndulo (7) es función de la gravedad ( $g$ ) y de la longitud de la cuerda $(l)$ :

$$
T=2 \pi \sqrt{\frac{l}{g}}
$$


Con la medición empírica de los períodos asociados a distintos cambios de longitud del péndulo, puedo estimar el valor de la gravedad $g$, linealizando el modelo y hallando la ecuación de regresión correspondiente.

\subsection{Estimación de un parámetro comparando dos modelos conocidos y acepadados come válidos}

Recordemos que la probabilidad de que ocurra un evento $P(A)$, se puede definir como:

$$
\begin{aligned}
& P(A)=\frac{\text { Casos Favorables }}{\text { Casos Posibles }} \\
& P(A)=\frac{\# \text { Total de Éxitos }}{\# \text { Total de Ensayos }}
\end{aligned}
$$

Si se inscribe una circunferencia en un cuadrado de lado un decímetro por ejemplo, y lanzo un objeto pequeño (un grano de arroz), la probabilidad de que éste caiga dentro de la circunferencia es:

$$
\begin{aligned}
& P(A)=\frac{\text { Área del Círculo }}{\text { Area del Cuadrado }} \\
& P(A)=\frac{\frac{\pi D^{2}}{4}}{L^{2}}
\end{aligned}
$$

Como $L=D$

$$
P(A)=\frac{\pi}{4}
$$

Ahora, si lanzo el objeto $N$ veces y en $n$ ocasiones de éstas cae dentro del círculo; usando la definición de probabilidad como frecuencia relativa se obtiene:

$$
P(A)=\frac{n}{N}
$$


Igualando estos dos modelos

$$
\frac{\pi}{4}=\frac{n}{N}
$$

Puedo estimar el valor del número $\pi$ con una aproximación sorprendente:

$$
\pi=\frac{4 n}{N}
$$

Un procedimiento análogo a éste fue utilizado por Ernest Rutherford cuando descubrió el diminuto núcleo atómico en su famoso experimento de la hoja de oro ${ }^{19}$.

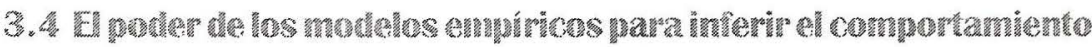

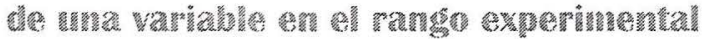

Un biólogo obtuvo un modelo que relaciona el número de bacterias por unidad de volumen presentes en un cultivo $(N)$, como función del tiempo $(t)$, tomando varios valores del tiempo entre 0 y 6 horas (rango experimental):

$$
N=32.14(1.43)^{t}
$$

El científico está convencido de que esta ecuación tiene un gran valor práctico para valores de $t$ entre 0 y 6 horas (no por fuera de este rango), porque obtuvo un valor muy alto del coeficiente de correlación.

Por ejemplo, se puede estimar el número de bacterias presentes por unidad de volumen 3.5 horas después de iniciado el experimento $(t=3.5)$.

$$
N=112
$$

Los cuatro ejemplos analizados en esta sección, se retomarán posteriormente con otros propósitos más concretos.

19 HEWitT / Robinson. Manual de laboratorio de física. México: 1998. P. 319, 320. 


\section{Conclusiones}

Considero que este primer acercamiento al estudio de los modelos: sus funciones (representación, mediación, artefactos epistémicos) y sus virtudes en el campo pragmático, cumple con los objetivos trazados al iniciar el artículo, porque se percibe el gran avance al reorientar la concepción axiomática de las teorías científicas, hacia las concepciones semánticas centradas en la noción de modelo.

Este cambio de concepción es el que ha permitido extender la noción de ley a otras ciencias como la psicología o la economía y, por tanto, estudiar los modelos utilizando herramientas de las ciencias cognoscitivas.

Los casos concretos de modelos que se han abordado, permiten reforzar las tesis propuestas al inicio del trabajo:

- Los modelos científicos no se pueden abordar desde una sola perspectiva, dada la multiplicidad de funciones que pueden cumplir y los distintos propósitos para los que se pueden utilizar.

“Este énfasis en la representación pone limitaciones excesivas en nuestra visión de los modelos. Los modelos deben pensarse más bien como artefactos epistémicos a través de los cuales nosotros adquirimos conocimiento de maneras diversas" (Knuutilla, pág. 1).

- Más que la propia discusión acerca de lo que es un modelo (artefacto epistémico, mediador, representación, componente de un sistema distribuido), se debe centrar la atención en el poder predictivo e inferencial de los modelos, que es lo que en última instancia los vuelve útiles para ser usados en la ciencia.

Sin necesidad de profundizar ni atacar la propuesta de los estructuralistas, Bas van Fraassen, Da Costa y French, he delimitado el campo de discusión a problemas concretos de la ciencia y he acotado el uso de herramientas matemáticas y estadísticas. 
En la línea de Giere ${ }^{20}$, me preocupa más el criterio para conectar el modelo abstracto con un sistema real, sin atribuirles una naturaleza matemática determinada. Se obvian los predicados conjuntistas, la noción de espacio de estados, el isomorfismo y el isomorfismo parcial.

\section{T2}

BUENO, Octavio. Empiricism, concervativeness and quasi - truth. En: http:// plato.stanford.edu/entries/rationalism-empiricism/ (Consultada diciembre 2005).

Scientific rationality and inconsistency: a partial structures prespective. En: http:// plato.stanford.edu/entries/rationalism-empiricism/ (Consultada diciembre 2005).

DIEGUEZ, Antonio. El realismo constructivo de Giere. Capítulo 7. Realismo científico. En: http://fs-morente.filos.ucm.es/publicaciones/revista/n24/ zamora.PDF (Consultada diciembre 2005).

DÍEZ, José A. y MOULINES, Ulices. Fundamentos de la filosofia de la ciencia. Barcelona: Ariel, 1999.

ECHAVARRÍA, Javier. Filosofia de la ciencia. Madrid: 1998. p. 75, 76.

FRAASSEN, Bas van. Science as representation: flouting the criteria. En: http:// philsci-archive.pitt.edu (Consultada diciembre 2005).

FRENCH, Esteven. A model-theoretic account of representation. En: http:// philsci-archive.pitt.edu (Consultada diciembre 2005).

FRIGG, Roman. Models and representation: why structures are not enough. En: http:// philsci-archive.pitt.edu philsci-archive.pitt.edu (Consultada diciembre 2005).

GIERE, Ronald N. Filosofia de la ciencia naturalizada. Traducción GONZÁLEZ GARCÍA, Marta.

La estructura congnitiva de las teorías científicas. Traducción GONZÁLEZ GARCÍA, Marta.

20 DÍEZ y MOULINES. 1999. p. 347. "Giere propone considerar las teorías como medios para definir modelos abstractos de los que se postula su aplicación a ciertos sistemas reales. Los modelos ahora no se caracterizan como entidades conjuntistas, ni mediante espacios de estados, ni de ninguna otra forma específica. No se les atribuye una naturaleza matemática determinada". 
How models are used to represent reality. 2004. p. 2, 6. En: http:// www.tc.umn.edu (Consultada diciembre 2005).

Models as parts of distributed cognitiva systems. En: http:// www. tc.umn.edu (Consultada diciembre 2005).

Models, methaphysics, and methodology. En: http:// www.tc.umn. edu (Consultada diciembre 2005).

GLENNAN, Stuart. A model of models. 2000. En: http:// philsci-archive.pitt. edu

HARTMANN, Stephan. Models as a tool for theory construction: some strategies of preliminary physics. En: http://philsci-archive.pitt.edu (Consultada diciembre 2005).

HENDRY, R. F. y PSILLOS, Statais. How to do thinks with theories: as interactive view of language and models in science. En: http:// www.oup.com/ us/catalog/general/subject/LiteratureEnglish/LiteraryTheory/? (Consultada diciembre 2005).

HEWITT / ROBINSON. Manual de laboratorio de física. México: 1998. p. 319, 320.

IBARRA, Andoni y MORMANN, Thomas. Representaciones en la ciencia: de la invariancia estructural a la significatividad pragmática. Barcelona: Ediciones del Bronce, 1997. 332 p.

JUSTUS, James. Qualitative scientific modeling and loop analysis. 9/13/04. En: http:// philsci-archive.pitt.edu (Consultada diciembre 2005).

KNUUTTILA, Tarja. A parser as epistemic artefact: a material view on models. En: http:// philsci-archive.pitt.edu (Consultada diciembre 2005).

MORRISON, Margaret. Models as representational structures. En: http:// / www.uni-konstanz.de/ppm/workshop2/Morrison_final.pdf (Consultada diciembre 2005).

PINCOCK, Chris. Overextending partial structures: idealization and abstraction. En: http:// philsci-archive.pitt.edu

SUÁREZ, Mauricio. An inferential conception of scientific representation. En: http:// philsci-archive.pitt.edu (Consultada diciembre 2005).

Scientific reprentation: against simlarity and isomorphism. 2003. En: http:// philsci-archive.pitt.edu (Consultada diciembre 2005).

VELASCO, Gabriel y WISNIEWSKI, Piots M. Probabilidad y estadística para ingeniería y ciencias. México: Thomson, 2002. p. 67, 68.

IV COLOQUIO internacional de filosofía e historia de las matemáticas. En: Ideas y valores, Universidad Nacional. Bogotá. Nos. 92 y 93; p. 80. 